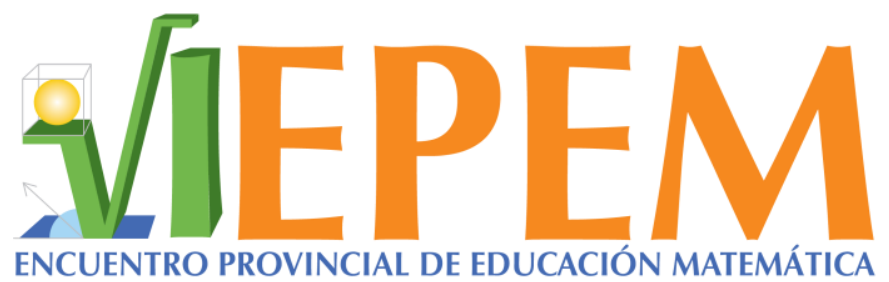

VI Encuentro Provincial de Educación Matemática.

27 al 29 de setiembre, 2017. Puntarenas, Costa Rica.

\title{
Desarrollo de habilidades de geometría analítica con Geogebra en décimo año
}

\author{
Erick Pizarro Carrillo \\ erick.pizarro.carrillo@una.cr \\ Universidad Nacional \\ Costa Rica. \\ Danny Ramírez Lobo \\ danny.ramirez.lobo@una.cr \\ Universidad Nacional \\ Costa Rica
}

\begin{abstract}
Resumen
En respuesta a la puesta en marcha de la Reforma Matemática en la educación costarricense se hace necesaria para los docentes la reformulación de sus lecciones. El uso inteligente de las tecnologías digitales es un eje fundamental en los programas de matemática, en este particular, el uso del computador o dispositivos móviles, para fomentar clases que sean dinámicas y significativas para los estudiantes.

La utilización del programa o aplicación de Geogebra para desarrollar las habilidades propuestas para geometría analítica, se basó en la idea de que el estudiantado pudiera argumentar, plantear y contrastar sus hipótesis, con la ayuda de la guía de trabajo y dialogando con sus compañeros.

Se logró con esta metodología tener estudiantes más activos, generando su propio aprendizaje, interesados por utilizar la tecnología en la clase y con mejor rendimiento académico.
\end{abstract}

Palabras clave: Enseñanza de la Matemática; Tecnología en el aula; Habilidades matemáticas; Geometría Analítica.

Experiencia de aula

Pizarro, E. y Ramírez, D. (2017). Desarrollo de habilidades de geometría analítica con Geogebra en décimo año. En Y. Morales-López, M. Picado, R. Gamboa, C. Martínez, M. Castillo y R. Hidalgo (Eds.), Memorias del VI Encuentro Provincial de Educación Matemática, Costa Rica, 2017 (pp. 1321). Heredia: Universidad Nacional. ISBN: 978-9968-9661-5-3. DOI: http://dx.doi.org/10.15359/epem.6.3 


\section{Introducción}

Las innovaciones que se han implementado por parte del Ministerio de Educación Pública en el sistema educativo costarricense en Matemática, en cuanto a los programas de estudio, han generado la necesidad de repensar las lecciones de esta asignatura. No solamente se han modificado los contenidos que se imparten en todos los niveles sino que se pretende cambiar la visión que culturalmente se le ha dado a la matemática, cambiar la forma de enseñar y aprender matemática (Morales-López, 2017). Para lograr esta renovación no solo se deben cambiar los contenidos sino también la metodología y las evaluaciones.

Garnier menciona en los Programas de estudio de Matemáticas para I, II y III Ciclos de la Educación General Básica y el Ciclo Diversificado que "la estrategia de los nuevos programas se propone fundamentar pedagógicamente el paso desde lo concreto hacia lo abstracto" también afirma que "se trata de desarrollar el rigor y la capacidad matemática para resolver problemas, para la aplicación, matematización y modelización matemática" (2012, p. 10); lo anterior da una pista de lo que se busca con la Reforma.

Esta pretensión de desarrollar habilidades matemáticas y no la de adquisición de contenidos hace que lecciones sean diferentes a lo que se ha estado trabajando durante muchos años en Costa Rica. Un currículo basado en procesos es más enriquecedor pues para Sánchez (2002) "los procesos de pensamiento son los componentes activos de la mente y por lo tanto son elementos básicos para construir, organizar y usar los conocimientos" (p. 18). Basado en eso, este trabajo, pretende potenciar los cinco procesos mencionados en los programas de estudio: argumentar y razonar, plantear y resolver problemas, conectar, representar, comunicar o expresar ideas; al utilizar el trabajo cotidiano del estudiante de forma tal que esas habilidades se logren en la clase con el esfuerzo creativo del discente.

Lo anterior hace notar una necesidad que se centra en modificación de la forma en la que se imparte la lección y en buscar fomentar en el estudiante mayor interés por la materia, y principalmente, un sentido crítico e involucrar activamente al joven en su aprendizaje. La mediación pedagógica es ahora un punto clave en el desarrollo de la lección y en la interacción entre el estudiante y el conocimiento o sus habilidades según Díaz y Hernández (2002) "la enseñanza corre a cargo del enseñante como su originador; pero al fin y al cabo es una construcción conjunta como producto de los continuos y complejos intercambios con los alumnos y el contexto instruccional” (p. 140)

Para Gamboa y Ballestero (2010) "las clases de geometría en la educación secundaria se han basado en un sistema tradicional de enseñanza, donde docentes presentan la teoría, desarrollan ejemplos y aportan los ejercicios que deben ser resueltos por estudiantes" (p.125). Consecuentemente no se manipulan los elementos geométricos y el uso de la tecnología queda de lado como herramienta de aprendizaje.

Por otra parte, para Guedez (2005) con el uso de software "se dinamizó el aula de clases; promoviendo en los estudiantes un sentido de autonomía en la adquisición de conocimientos" (p.49) una de las principales ideas de los nuevos programas y en esta 
Desarrollando habilidades de geometría analítica con Geogebra en décimo año

experiencia, lo cual permite lograr que las lecciones sean más atractivas y se fortalezca el alcance de las habilidades mediante la utilización de la herramienta informática.

\section{Descripción de la experiencia de aula}

A continuación se indican las habilidades específicas que se trabajaron con la puesta en práctica de la situación de aprendizaje planteada durante seis lecciones, según se mencionan en los programas de estudios de Matemática (2012, p.386)

- Representar gráficamente una circunferencia dado su centro y su radio.

- Representar algebraicamente una circunferencia dado su centro y su radio.

- Aplicar traslaciones a una circunferencia.

- Resolver problemas relacionados con la circunferencia y sus representaciones.

- Determinar gráfica y algebraicamente si un punto se ubica en el interior o en el exterior de una circunferencia.

- Determinar si una recta dada es secante, tangente o exterior a una circunferencia

Los contenidos que se abarcan son ecuación canónica de la circunferencia, traslación de circunferencias, puntos interiores, exteriores y sobre la circunferencia, rectas secantes, rectas tangentes y rectas exteriores.

Para llevar a cabo esta experiencia fue necesario contar con algunos materiales tales como la Tablet, computadora, Smartphone (dispositivo móvil) o laboratorio informático. Además de la guía de trabajo que los estudiantes al desarrollarla, puedan alcanzar las habilidades matemáticas anteriormente indicadas. Con esta guía se independiza el trabajo el estudiante.

La experiencia de aula se llevó a cabo con los estudiantes de un Colegio Público diurno de Heredia. Primeramente se les indicó a los estudiantes que se trabajará de forma diferente durante las siguientes seis lecciones. Una vez que se cuenta con la herramienta tecnológica se procede a descargar el programa o aplicación Geogebra y a su vez que los educandos inicien la manipulación de la aplicación durante 15 minutos.

Después que el estudiante se familiariza con la aplicación, interviene nuevamente el docente, que debe ser ayuda para el uso de la interfaz así como mediador de la actividad matemáticamente, para las dudas que el estudiante vaya generando, propiamente sobre los resultados algebraicos que desplegará el programa. El docente no debe brindar una respuesta, sino que debe responder de forma que le permita al estudiante reflexionar y razonar acerca la cuestión planteada, poniendo a prueba sus hipótesis y fomentando el diálogo entre ellos. 
Desarrollando habilidades de geometría analítica con Geogebra en décimo año

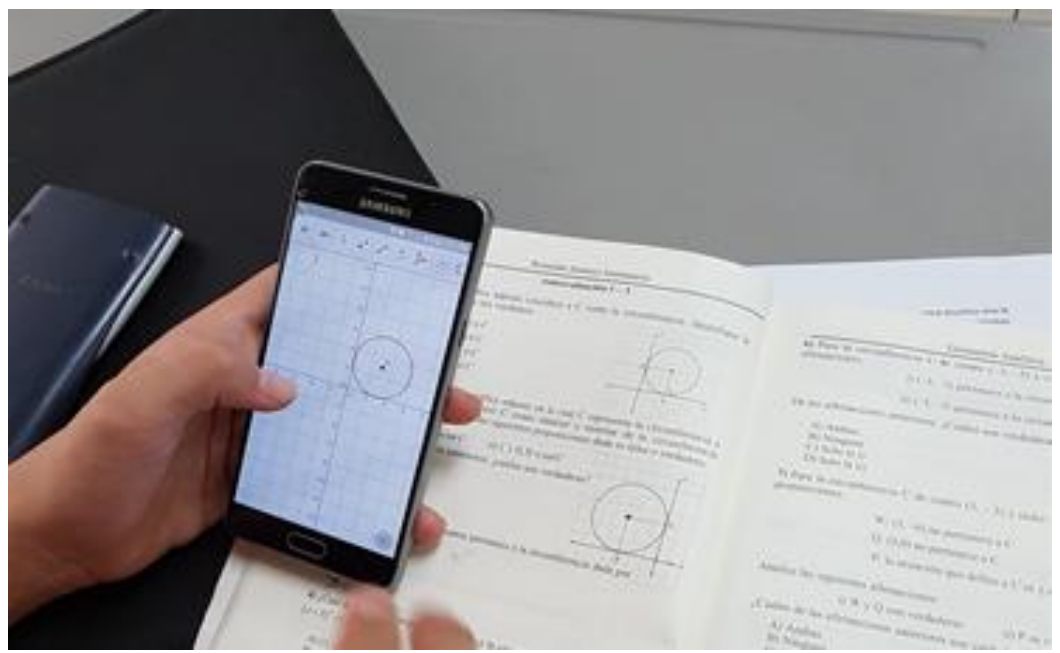

Figura 1. Manipulación de la aplicación

Durante el desarrollo de la guía, en una primera etapa, se le solicita al estudiante construir circunferencias con un centro y radio indicado, trasladar circunferencias e ir anotando el resultado algebraico que despliega el programa, luego se le plantean algunas interrogantes como ¿Qué modificaciones se dan en la expresión algebraica al realizar diferentes movimientos? ¿Qué se mantiene invariante?, para que pueda observar relaciones, realizar hipótesis y concluir algunos resultados.

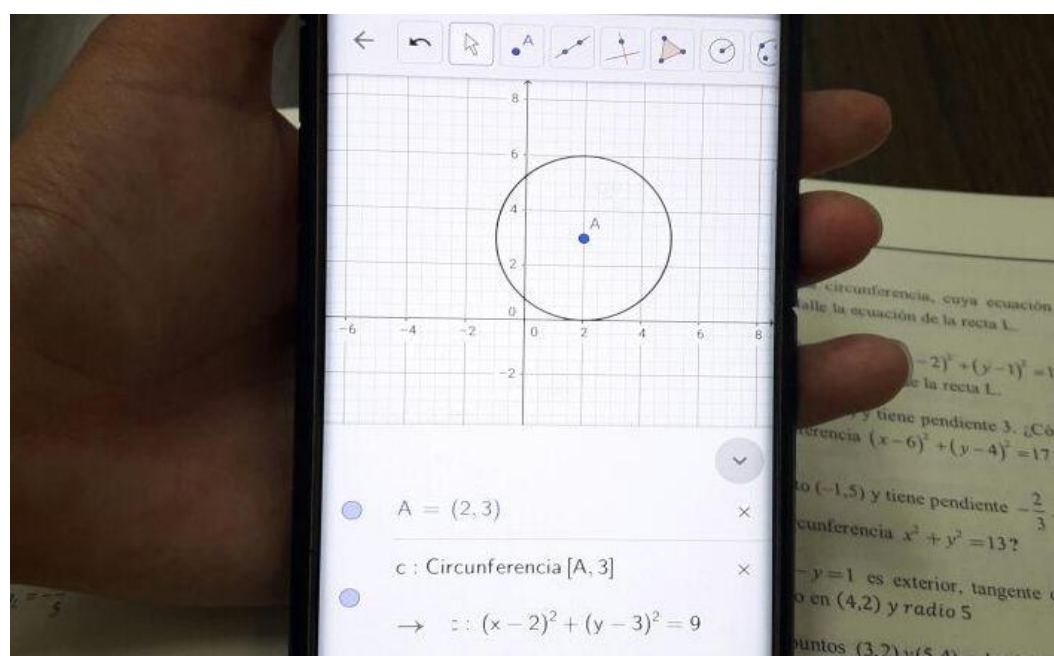

Figura 2. Trabajo de aula

En una segunda etapa, donde el estudiante ya ha trabajado las primeras tres habilidades y con un mayor dominio de la aplicación, se procede con el planteamiento de un problema donde pueda aplicar las habilidades adquiridas que le permitan construir nuevos conceptos como punto interior, exterior y sobre la circunferencia así como las rectas secantes, tangentes y exteriores. 
Desarrollando habilidades de geometría analítica con Geogebra en décimo año

Finalmente, se realiza un cierre la actividad donde el profesor, en conjunto con los discentes, establece los conceptos con la rigurosidad matemática necesaria.

Por otra parte, en lo que concierne a la Evaluación, se brinda una actividad evaluativa donde el estudiante, utilizando la aplicación, pueda darle solución a los diferentes ejercicios planteados, considerando niveles de dificultad que le permitan lograr las habilidades planteadas al inicio del documento.

\section{Resultados obtenidos}

Lo que inició como una necesidad pedagógica para una lección de geometría, la creación de una unidad didáctica para trabajar con los contenidos de geometría analítica en décimo año, se convirtió en toda una experiencia de aprendizaje tanto para los docentes involucrados como para los estudiantes del colegio seleccionado. Al ver el éxito que se alcanzó con esta propuesta se deseó compartir con la comunidad docente del país y hacer la socialización de la misma.

Partiendo de esto se logró realizar la unidad didáctica para una clase exitosa en varios ámbitos tanto para el docente como para el discente, planificar una lección en la que el uso de la tecnología y el aprendizaje significativo estuviese presente de principio a fin.

Al manipular el programa y tener la representación algebraica y ver sus cambios, razonaron, plantearon y argumentaron sus hipótesis para comunicarlas de forma escrita y verbal con sus compañeros y docente, además lograron representar en forma gráfica con ayuda del Geogebra y conectar la relación entre las representaciones, cumpliendo en gran medida, los cinco procesos planteados por el Ministerio de Educación Pública en sus programas de estudio.

Un gran aporte de esta dinámica de la clase fue que los estudiantes salieron de la rutina de escribir, ver o resolver en el cuaderno. El simple uso del teléfono inteligente o de la computadora hace que ellos se sientan más interesados en la materia.

Esta implementación se les hace natural pues ellos manipulan y viven con esta tecnología a la mano, literalmente, son nativos digitales y esto se aprovechó para su aprendizaje. Dado lo anterior se tuvo en el aula más interesada y con mejor disposición para el aprendizaje de la matemática, lo vieron como un juego y notaron hasta después que estaban aprendiendo, razonando y dialogando conocimientos matemáticos, pero no de forma memorística sino de forma amena y hasta divertida.

Esta actividad mejoró el trabajo cotidiano del estudiante en el aspecto cualitativo y cuantitativo, estuvieron atentos a la clase y lograron completar las actividades propuestas.

Una gran innovación es el uso inteligente de la tecnología, se pudo incorporar esta valiosa herramienta en la clase, como lo piden los programas, sobre todo en el aprendizaje desarrollo de las habilidades matemáticas.

Los beneficios no solo fueron cualitativos como los logros anteriores, sino que cuantitativamente también se obtuvieron buenos resultados en esta experiencia, basados en los resultados del trabajo cotidiano de la estrategia de evaluación.

VI Encuentro Provincial de Educación Matemática, Costa Rica, 2017. 
Desarrollando habilidades de geometría analítica con Geogebra en décimo año

\section{Referencias}

Ballestero, E. y Gamboa, R. (2010). La enseñanza y aprendizaje de la geometría en secundaria, la perspectiva de los estudiantes. Revista Electrónica Educare, 14(2), 125-142. Recuperado de https://dialnet.unirioja.es/descarga/articulo/5414933.pdf

Díaz, F. y Hernández, G. (2002). Estrategias docentes para un aprendizaje significativo: Una interpretación constructivista (2a ed.). México, D.F.: McGraw-Hill Interamericana.

Guedez, M. (2005). El aprendizaje de funciones reales con el uso de un software educativo: una experiencia didáctica con estudiantes de educación de la ULA-Táchira. Revista Acción Pedagógica, 14, 38-49. Recuperado de https://dialnet.unirioja.es/descarga/articulo/2968990.pdf

Ministerio de Educación Pública de Costa Rica (2012). Programas de estudio Matemáticas. I, II y III Ciclos de la Educación General Básica y Ciclo Diversificado. Costa Rica: autor.

Morales-López, Y. (2017). Costa Rica: The Preparation of Mathematics Teachers. En A. Ruiz (Ed.), Mathematics Teacher Preparation in Central America and the Caribbean (pp. 39-56). Springer International Publishing. Recuperado de http://link.springer.com/chapter/10.1007/978-3-319-44177-1_3

Sánchez, M. (2002). La investigación sobre el desarrollo y la enseñanza de las habilidades del pensamiento. Revista Electrónica de Investigación 4(1). Recuperado de http://redie.ens.uabc.mx/vol4no1/contenido-amestoy.html 


\section{Anexo 1}

\section{Guía de trabajo individual}

Indicaciones generales: Construya lo que se le solicita en cada sección, si tiene alguna duda puede consultar con su docente.

\section{Sección 1:}

1. Inicie el programa Geogebra desde su dispositivo

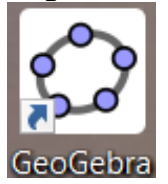

2. En la barra de herramientas seleccione CIRCUNFERENCIA (centro, radio)

\section{() Circunferencia (centro, radio)}

Trace la circunferencia con centro $A(1,3)$ y radio $r=3$.

Escriba el resultado de la vista algebraica:

3. Seleccione ELIGE Y MUEVA de la barra de herramientas

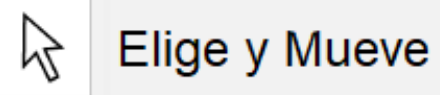

Mueva la circunferencia a los diferentes cuadrantes

Escriba algunos de los resultados de la vista algebraica:

¿Qué se modificó? ¿Qué no se modificó? ¿Qué relación puede notarse entre la representación gráfica y la representación algebraica?

Mueva la circunferencia colocando el centro sobre uno de los ejes de coordenadas, y luego sobre el origen

Escriba algunos de los resultados de la vista algebraica:

¿Qué se modificó? ¿Qué no se modificó? ¿Qué relación puede notarse entre la representación gráfica y la representación algebraica? 
Desarrollando habilidades de geometría analítica con Geogebra en décimo año

Sección 2:

1. Inicie el programa Geogebra desde su dispositivo

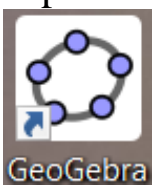

2. En la barra de herramientas seleccione CIRCUNFERENCIA (centro, radio)

\section{(-) Circunferencia (centro, radio)}

Trace la circunferencia con centro $A(1,4)$ y radio $r=4$.

En la barra de herramientas seleccione

\section{A Punto}

Trace los puntos $B(-3,6), \quad C(5,4), \quad D(2,3)$

En la barra de herramientas selecciones

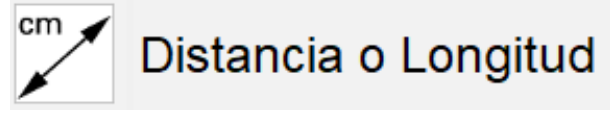

Calcule la medida de los $\overline{A B}=$ ,$\overline{A C}=$ $\overline{A D}=$

Compare estas medidas con el radio de la circunferencia.

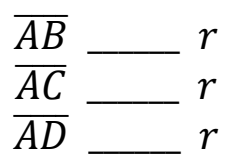

Clasifique los puntos B, C y D como interior, exterior o sobre la circunferencia

Sección 3:

1. Inicie el programa Geogebra desde su dispositivo

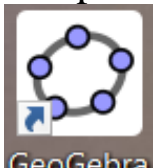

2. En la barra de herramientas seleccione CIRCUNFERENCIA (centro, radio)

\section{(-) Circunferencia (centro, radio)}

Trace la circunferencia con centro $A(1,1)$ y radio $r=2$.

En la barra de entrada

\section{Entrada:}

VI Encuentro Provincial de Educación Matemática, Costa Rica, 2017. 
Desarrollando habilidades de geometría analítica con Geogebra en décimo año

Trace las rectas $y=3, y=-x+1, y=x+3$

Calcule cuántas intersecciones tienen estas tres rectas con la circunferencia.

Clasifique las rectas como secante, exterior o tangente a la circunferencia

$y=3$

$y=-x+1$

$y=x+3$

Sección 4:

Resuelva la siguiente situación

Un barco de rescate se encuentra en alta mar en la búsqueda de una persona. Supongamos que dicha persona está en el origen $(0,0)$, y el barco se encuentra $50 \mathrm{~km}$ al norte y $40 \mathrm{~km}$ al oeste. El capitán del barco, con su telescopio, tiene un radio de visión de $20 \mathrm{~km}$. ¿Podrá visualizar en este momento a la persona?

Si el barco se traslada $80 \mathrm{~km}$ al sur, luego $50 \mathrm{~km}$ al este. Entonces ¿se visualiza la persona desde este punto?

Si se desplaza ahora $20 \mathrm{~km}$ al norte ¿Logrará divisar el punto en alta mar donde se encuentra la persona?

¿A qué distancia se encuentra el barco de la persona a rescatar?

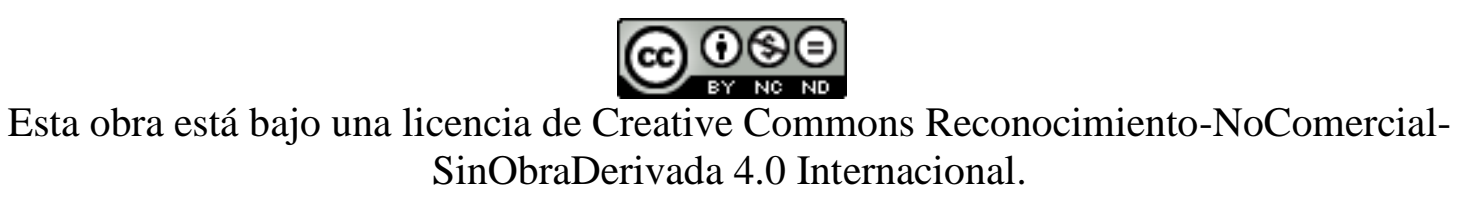

VI Encuentro Provincial de Educación Matemática, Costa Rica, 2017. 